\title{
NUMERICAL SIMULATION OF SUBGRID-SCALE MODEL FOR 2D TURBULENCE
}

\author{
Surajate BOONYA-AROONNET ${ }^{1}$ and Yasuyuki SHIMIZU 2 \\ ${ }^{1}$ Student Member of JSCE, Ph.D. Student, Dept. of Hydro science \& Environmental Engineering, \\ Hokkaido University (North 13, West 8, Kita-ku, Sapporo, Hokkaido 060-8628, Japan) \\ 2 Member of JSCE, Dr. of Eng., Associate Professor, Dept. of Hydro science \& Environmental Engineering, \\ Hokkaido University (North 13, West 8, Kita-ku, Sapporo, Hokkaido 060-8628, Japan)
}

\begin{abstract}
Presently the simulation of horizontal turbulent motions in the flows is feasible and it reduces the dependence on turbulence-closures applied in URANS i.e. simulating 2D-turbulent motions with a dominant vertical vorticity component, called Large Eddy Simulation (LES). The idea is that kinetic energy has to be conserved because the part of the kinetic energy cascaded towards smaller scales of eddy movement requires an energy-controlling subgrid-scale (SGS) model. To connect this unresolved part of 2D-turbulent motions, the additional SGS model is coupled with our existing 2D model by modification of eddy viscosity. Hence the calculated results are compared with our experiment. The SGS model has been successfully applied to simulating a flow ficld and shows an improvement to our existing model based on experimental data especially at area of high turbulent fluctuation.
\end{abstract}

Key Words: LES, Numerical Simulation, SGS, 2D-turbulence, URANS

\section{INTRODUCTION}

Recently in flow simulations, the Large Eddy Simulation (LES) is receiving more interests in simulation of 2D turbulence. Unlike 3D turbulence, where large swirling flow structures break up into little ones, as can be seen in every plume of smoke, in 2D turbulence, small-scale structures surprisingly merge to bigger ones. An initially disorganized 2D flow eventually becomes organized in large persistent vortex structures.

Briefly, LES seeks to predict the motion of the largest and most important eddies uncoupled from the small eddies. This uncoupling is important because the large eddies are resolvable on a computational mesh only. The idea of LES is that the effects of large scales are directly computed, and only the small subgrid-scales are modeled. For ocean-scale simulation of eddies, the entropy balance is prominent which demands control over entropy such as by the kinetic-energy conserving Subgrid Scale $(\mathrm{SGS})^{1)}$. For smaller-scale simulation of flows for example near the river bank - locally has stronger turbulent fluctuation because of existence of river structures, or other topographic features (islands, headlands, jetties, groins, etc.) leading to a local flow separation ${ }^{2)}$ - the part of the kinetic energy cascaded towards smaller scales requires an energy-controlling SGS model.

To improve assessment of navigation, structural stability, sediment transport, dredging operations, oil spills, algae blooms etc., there appears a growing interest in simulating more details of the flow.

The classical approach would be to follow the concepts of unsteady Reynolds-Averaged Navier Stokes (URANS) computations and accordingly design a closure for 2D-turbulence. The design of an adequate closure for 2D-turbulence is a complicated task due to the multiplicity of internal and external sources that induce or supply energy to 2D-turbulence. Inevitably, unknown coefficients appear that require calibration for which detailed observations are scarce. A particular type of turbulence closure is required for representing the action of the unresolved part of 2D-turbulence. The latter turbulence closure is called SGS model as it depends on low-pass filtering of the physical flow by the grid size.

In this study, a numerical 2D flow model with a Moving Boundary Fitted Coordinate ${ }^{3)}$ (MBFC) 
originated from 2D continuity and momentum equations are used in this analysis. In addition, finite difference method is employed to numerically calculate the modified flow equations with a high-order Godunov scheme known as the Cubic Interpolated Pseudoparticle (CIP) Method ${ }^{4}$. Based on the available model, the additional SGS model is coupled in order to answer the question that whether the available model is suitable for LES to simulate the 2D-turbulent motions or not. In this case study, the turbulence generated from river structures in a meandering river was selected. The simulated results are compared between two cases, with \& without SGS models. The computational results are also verified with our available physical model experiment ${ }^{5)}$. Further, the influence of mesh size to the SGS contributions to eddy viscosity is also investigated.

Therefore, this paper deals with these questions and examines the possibility of using the SGS model to the 2D-turbulence flow model in the case of meandering river with river structures that generate turbulence. These are the motivations to model the eddy viscosity in more details by SGS model. The next section reviews the equation and SGS model applied in this study.

\section{FLOW EQUATIONS}

In order to simulate the flow, the $2 \mathrm{D}$ continuity and momentum equations are used as starting point.

$$
\begin{aligned}
& \frac{\partial h}{\partial t}+\frac{\partial(h u)}{\partial x}+\frac{\partial(h v)}{\partial y}=0 \\
& \frac{\partial(h u)}{\partial t}+\frac{\partial\left(h u^{2}\right)}{\partial x}+\frac{\partial(h u v)}{\partial y}=-C_{d} u \sqrt{u^{2}+v^{2}}- \\
& g h \frac{\partial H}{\partial x}+\left(\frac{\partial}{\partial x}\left\{v_{t} \frac{\partial(u h)}{\partial x}\right\}+\frac{\partial}{\partial y}\left\{v_{t} \frac{\partial(u h)}{\partial y}\right\}\right) \\
& \frac{\partial(h v)}{\partial t}+\frac{\partial(h u v)}{\partial x}+\frac{\partial\left(h v^{2}\right)}{\partial y}=-C_{d} v \sqrt{u^{2}+v^{2}}- \\
& g h \frac{\partial H}{\partial y}+\left(\frac{\partial}{\partial x}\left\{v_{t} \frac{\partial(v h)}{\partial x}\right\}+\frac{\partial}{\partial y}\left\{v_{t} \frac{\partial(v h)}{\partial y}\right\}\right)
\end{aligned}
$$

where $\mathrm{x}, \mathrm{y}$ are co-orthogonal coordinates; $\mathrm{t}$ is time; $\mathrm{u}, \mathrm{v}$ are depth-averaged velocity components in $\mathrm{x}, \mathrm{y}$ directions respectively; $h$ is depth; $H$ is water surface elevation; $g$ is gravitational acceleration; and $C_{d}$ is the friction coefficient. The eddy viscosity $v_{t}$ will be explained later in Section 3. The $(x, y, t)$ coordinate system, employed in above equations, is

transformed into $(\xi, \eta, \tau)$ coordinates, Moving Boundary Fitted Coordinate (MBFC) system ${ }^{3)}$. The continuity and momentum equations of the flow, (2.1) to (2.3) in the MFBC are eventually rewritten here.

$$
\frac{\partial}{\partial \tau}\left(\frac{h}{J}\right)+\frac{\partial}{\partial \xi}\left[\left(\xi_{t}+u^{\xi}\right) \frac{h}{J}\right]+\frac{\partial}{\partial \eta}\left[\left(\eta_{t}+u^{\eta}\right) \frac{h}{J}\right]=0
$$$$
\frac{\partial u^{\xi}}{\partial \tau}+\left(\xi_{t}+u^{\xi}\right) \frac{\partial u^{\xi}}{\partial \xi}+\left(\eta_{t}+u^{\eta}\right) \frac{\partial u^{\eta}}{\partial \eta}
$$

$+\alpha_{1} u^{\xi} u^{\xi}+\alpha_{2} u^{\xi} u^{\eta}+\alpha_{3} u^{\eta} u^{\eta}-D_{\xi}$

$=-g\left[\left(\xi_{x}^{2}+\xi_{y}^{2}\right) \frac{\partial H}{\partial \xi}\left(\xi_{x} \eta_{x}+\xi_{y} \eta_{y}\right) \frac{\partial H}{\partial \eta}\right]$

$-\frac{C_{d} u^{\xi}}{h J} \sqrt{\left(\eta_{y} u^{\xi}-\xi_{y} u^{\eta}\right)^{2}+\left(-\eta_{x} u^{\xi}-\xi_{x} u^{\eta}\right)^{2}}$

$\frac{\partial u^{\eta}}{\partial \tau}+\left(\xi_{t}+u^{\xi}\right) \frac{\partial u^{\eta}}{\partial \xi}+\left(\eta_{t}+u^{\eta}\right) \frac{\partial u^{\eta}}{\partial \eta}$

$+\alpha_{4} u^{\xi} u^{\xi}+\alpha_{5} u^{\xi} u^{\eta}+\alpha_{6} u^{\eta} u^{\eta}-D_{\eta}$

$=-g\left[\left(\eta_{x}^{2}+\eta_{y}^{2}\right) \frac{\partial H}{\partial \eta}\left(\xi_{x} \eta_{x}+\xi_{y} \eta_{y}\right) \frac{\partial H}{\partial \xi}\right]$

$-\frac{C_{d} u^{\eta}}{h J} \sqrt{\left(\eta_{y} u^{\xi}-\xi_{y} u^{\eta}\right)^{2}+\left(-\eta_{x} u^{\xi}-\xi_{x} u^{\eta}\right)^{2}}$

In MBFC system, the $\xi, \eta$ are spatial coordinates and $\tau$ is time; $\mathrm{D}_{\xi}, \mathrm{D}_{\eta}$ are momentum diffusion term in $\xi, \eta$ directions. Moreover, other coefficients are described here.

$$
\begin{aligned}
& \alpha_{1}=\xi_{x} \frac{\partial^{2} x}{\partial \xi^{2}}+\xi_{y} \frac{\partial^{2} y}{\partial \xi^{2}} \\
& \alpha_{2}=2\left(\xi_{x} \frac{\partial^{2} x}{\partial \xi \partial \eta}+\xi_{y} \frac{\partial^{2} y}{\partial \xi \partial \eta}\right) \\
& \alpha_{.3}=\xi_{x} \frac{\partial^{2} x}{\partial \eta^{2}}+\xi_{y} \frac{\partial^{2} y}{\partial \eta^{2}} \\
& \alpha_{4}=\eta_{x} \frac{\partial^{2} x}{\partial \xi^{2}}+\eta_{y} \frac{\partial^{2} y}{\partial \xi^{2}} \\
& \alpha_{5}=2\left(\eta_{x} \frac{\partial^{2} x}{\partial \xi \partial \eta}+\eta_{y} \frac{\partial^{2} y}{\partial \xi \partial \eta}\right) \\
& \alpha_{6}=\eta_{x} \frac{\partial^{2} x}{\partial \eta^{2}}+\eta_{y} \frac{\partial^{2} y}{\partial \eta^{2}}
\end{aligned}
$$




$$
\begin{aligned}
& D_{\xi}=\frac{\partial}{\partial \xi}\left(v_{t} \xi_{r}{ }^{2} \frac{\partial u^{\xi}}{\partial \xi}\right)+\frac{\partial}{\partial \eta}\left(v_{t} \eta_{r}{ }^{2} \frac{\partial u^{\xi}}{\partial \eta}\right) \\
& D_{\eta}=\frac{\partial}{\partial \xi}\left(v_{t} \xi_{r}{ }^{2} \frac{\partial u^{\eta}}{\partial \xi}\right)+\frac{\partial}{\partial \eta}\left(v_{t} \eta_{r}{ }^{2} \frac{\partial u^{\eta}}{\partial \eta}\right)
\end{aligned}
$$

However, by applying above described equations, the eddy movement smaller than mesh size remains unresolved. Therefore, the smaller eddy is further modeled in the succeeding section.

\section{SUBGRID-SCALE MODEL}

The prediction of the flow motion is coupled by the equations in the previous section. The large eddy motions are resolved on a computational mesh. For smaller eddy motions, the eddy viscosity is modeled in this section in order to control the energy for smaller scale movements. Thus, the subgrid scale is modeled for the horizontal component of the subgrid eddy viscosity, as well. Finally, we define the eddy viscosity $v_{t}$ in previous section by;

$v_{t}=v_{G}+v_{S G S}$

where $v_{G}$ and $v_{S G S}$ are eddy viscosity of grid scale and subgrid scale. The eddy viscosity of grid scale is described by parabolic eddy viscosity in a logarithmic velocity profile.

$v_{G}=\frac{1}{6} \kappa u_{*} h$

where $u_{*}$ is shear velocity; $\kappa$ is von Karman constant. For SGS model,

$v_{S G S}=\frac{1}{k_{s}^{2}}\left(\sqrt{\left(\gamma \sigma_{T} S^{*}\right)^{2}+B^{2}}-B\right)$

where,

$$
\begin{aligned}
& B=\frac{3}{4} \frac{g|\underline{U}|}{H C^{2}} \\
& \gamma=0.442 \sqrt{1-\alpha^{-2}} \\
& \sigma_{T}=\frac{u \Delta t}{\Delta x} \\
& \left(S^{*}\right)^{2}=2\left(\frac{\partial u^{*}}{\partial x}\right)^{2}+2\left(\frac{\partial v^{*}}{\partial y}\right)^{2}+\left(\frac{\partial u^{*}}{\partial y}\right)^{2}+ \\
& \left(\frac{\partial v^{*}}{\partial x}\right)^{2}+2 \frac{\partial u^{*}}{\partial y} \frac{\partial v^{*}}{\partial x}
\end{aligned}
$$

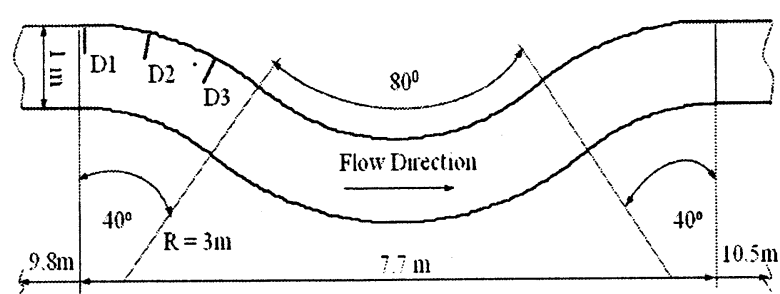

Fig.1 Flume layout and location of dikes

where $k_{s}$ is equivalent roughness height; $\sigma_{T}$ is Courant number; $C$ is Chezy coefficient; $\alpha$ is slope in the log-log spectrum of energy density. In (3.7), the asterisk $\left(^{*}\right)$ denotes fluctuating flow variables and these are defined through the recursive high-pass filter operator ${ }^{6)}$. The other parameter values in SGS model are set according to recommended values ${ }^{7}$.

\section{EXPERIMENT}

The experiment ${ }^{5}$ was conducted in laboratory flume of Applied Hydrology Laboratory of Hokkaido University. A $28-\mathrm{m}$ long and $1 \mathrm{~m}$ wide rectangle flume with a meandering reach of $7.7 \mathrm{~m}$ wave length with 3 consecutive bends of $40^{\circ}, 80^{\circ}$, $40^{\circ}$ arc angles respectively (Fig. 1 ) was constructed.

Three non-submerged spur dikes were used $(\mathrm{h}=15 \mathrm{~cm})$. The length is $1 / 4$ of channel width $(25 \mathrm{~cm})$ and spacing is 3 times spur's length $(75 \mathrm{~cm})$. Experiment was conducted for four cases i.e. Case1: without spur dikes; Case2: one spur dike was placed at the radial distance of $1^{\circ}$ from the bend entrance (concave part); Case3: two spur dikes were placed at $1^{\circ}, 15^{\circ}$; Case 4: three spur dikes were placed at $1^{\circ}$, $15^{\circ}$ and $29^{\circ}$ respectively. The experimental condition has been adopted according to the characteristics of Plane Rivers with mild slope and sub-critical flow. The experimental condition has been summarized in Table1.

\section{Table 1 Flow Conditions}

\begin{tabular}{lr}
\hline Discharge $\left(\mathrm{m}^{3} / \mathrm{s}\right)$ & 0.02 \\
Approach flow depth, $\mathrm{d}(\mathrm{m})$ & 0.095 \\
Average velocity, $\mathrm{U}_{\mathrm{o}}(\mathrm{m} / \mathrm{s})$ & 0.21 \\
Channel slope (Slope of platform) & $1: 2500$ \\
\hline
\end{tabular}

With the configurations and conditions explained in this section, the calculation was set up and the results are then discussed in the next section. 


\section{RESULTS AND DISCUSSION}

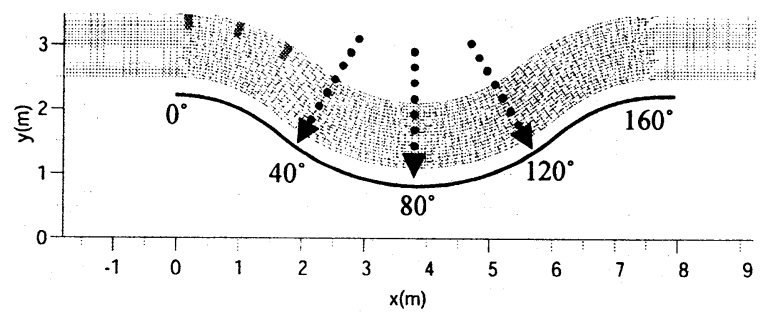

Fig.2 The calculation grid generated to simulate the flow.
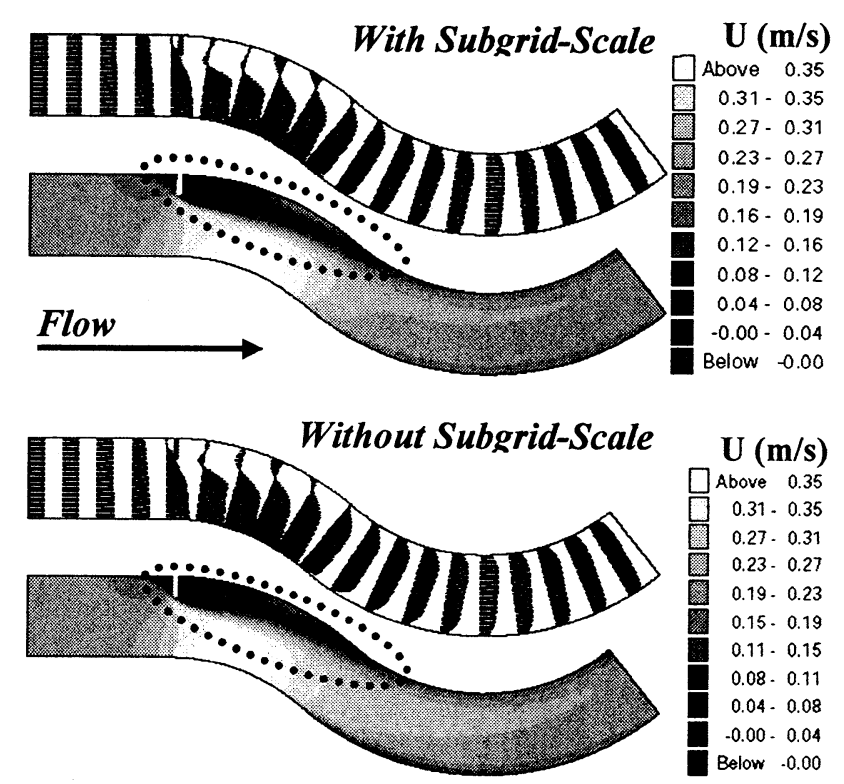

Fig. 3 Time-averaged velocity $\left(50^{\text {th }}-100^{\text {th }} \mathrm{sec}\right.$ interval $)$ comparing two cases (with and without Subgrid-Scale).

In this study, the simulations were performed only in two cases, Casel and Case 4 according to the previously described experiment section. Firstly, the calculation grid (Fig.2) was generated (approximately $2 \times 2 \mathrm{~cm}$ totally, $277 \times 50$ grids). The simulation period is 100 seconds and to avoid an influence from the warming up period of calculation, all time-averaged velocities are averaged over the second half of $50^{\text {th }}-100^{\text {th }}$.

Based on the time-averaged value, the calculated $\mathrm{u}$ is plotted in Fig.3 to compare the difference between two cases i.e. with and without SGS model. It is observed that the difference is around the area behind the spur dike, a recirculation zone separated by a spur dike. If we have a closer look at this area (behind the dike having negative velocity) displayed in Fig. 4 showing a cross-sectional velocity profile, the results from simulation with SGS model will give closer values to the experiment results. This proves that the additional SGS model into modification of eddy viscosity to calculate the turbulence suitably gives better results to our existing 2D model.
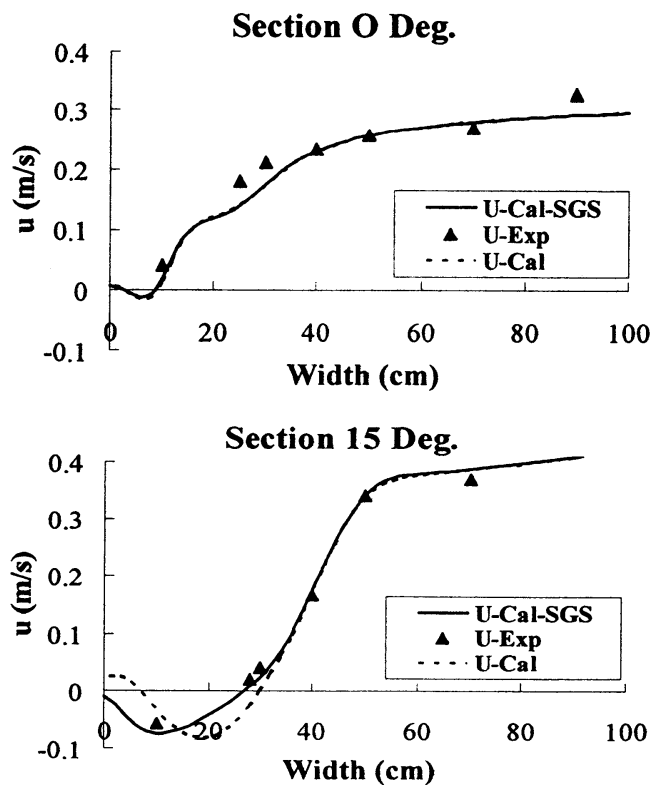

Fig.4 Plot of selected cross-sectional velocities, calculated with case of one dike located at $0^{\circ}$.

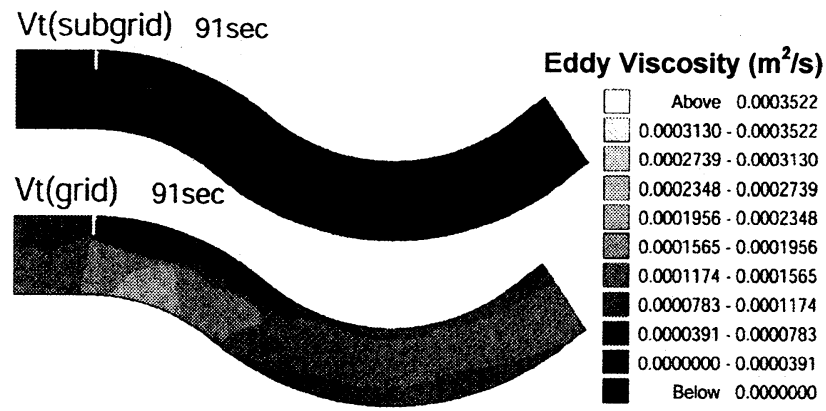

Fig.5 Eddy viscosity from calculation, above picture is an extra $v_{T}$ from SGS model.

However, the results form Fig. 4 show that the SGS can improve the results only in the flow separation zone, while there is no improvement to the area out of flow separation or the area of further downstream having positive velocity (the solid line and detached line in Fig.4 go the same way). Thus, to yield more accurate results, SGS becomes more significant in the case of stronger turbulent flow i.e. flow separation zone in our case. The reason could be that in this zone, the energy in momentum equation has to be balanced by adding SGS model. To emphasize this point (see Fig.5), it can be noticed that the SGS model contributes additional eddy viscosity only in the separation zone.

To further verify our exiting model that it is suitable for simulating LES, another simulation was performed in the case of all three dikes placed together and the results are shown in Fig.6. and Fig.7 


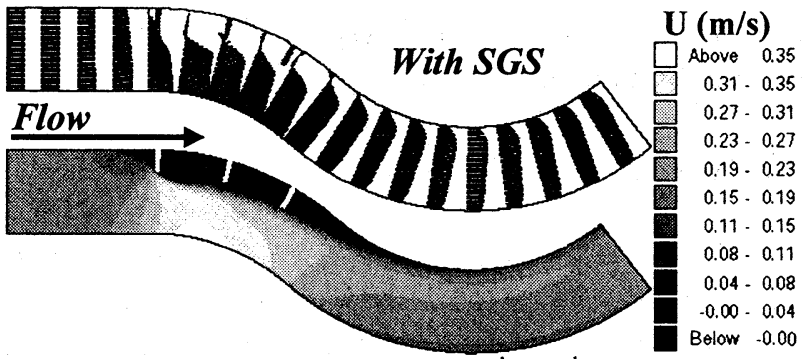

Fig.6 Time averaged-velocities $\left(50^{\text {th }}-100^{\text {th }} \mathrm{sec}\right.$ interval $)$ in the case of three dikes existing in the model.
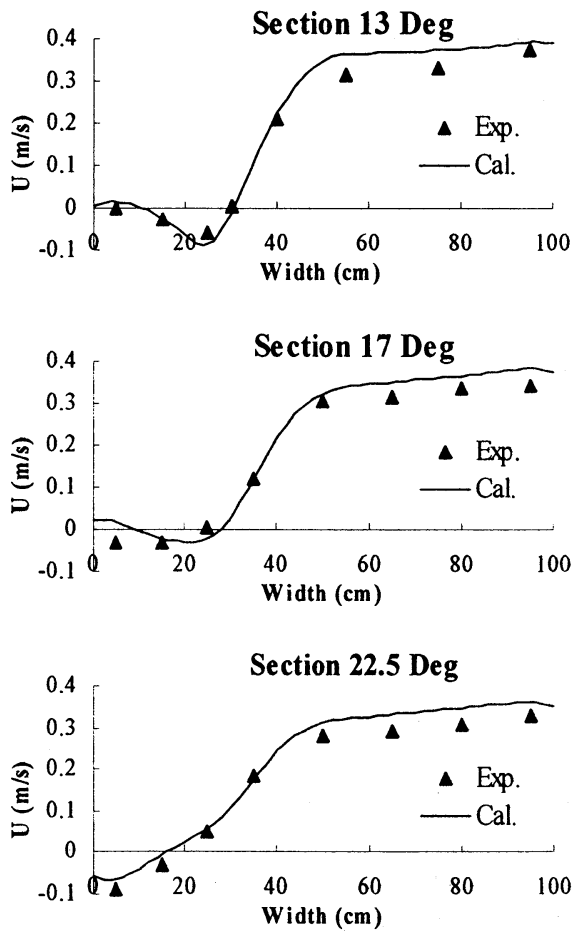

Fig.7 Plot of selected cross-sectional velocities, calculated with case of three dikes existing at location of $0^{\circ}, 15^{\circ} \& 29^{\circ}$.

A similar recirculation zone can be observed form Fig.6 compared to the case of only one dike, however it is slightly larger because the second and the third dikes placed within the recirculation zone generated by the first dike. The velocity plots are shown as well in Fig.7 for various locations of sections in the case of three dikes. These results of calculation also gave a good agreement to the experiment in the case of three dikes. Therefore, it can be said that our existing 2D model together with LES can be suitably applied to simulate the turbulence and eddy movements in the flow with additional SGS model. In addition, a plot of simulated eddy viscosity is also shown here in Fig.8.

It is also very interesting to visualize the coherent structures or vortices occurring in our simulation for a future analysis since the vortex is an important phenomenon in hydrodynamics e.g. shear stress amplification or in environmental point of view for mixing processes. The vortices appear at the same place as the flow separation zone since this area has a stronger turbulent fluctuation (Fig.9).

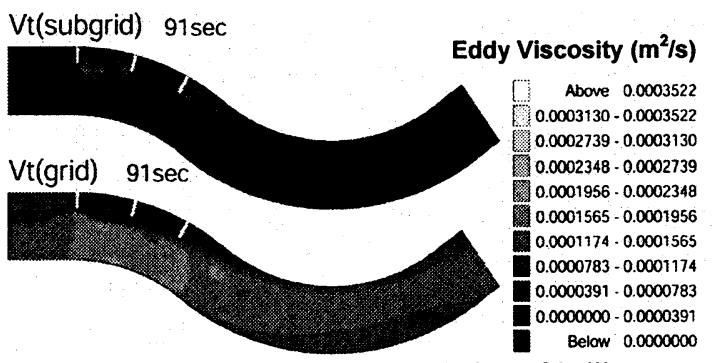

Fig. 8 Eddy viscosity from calculation of 3 dikes case

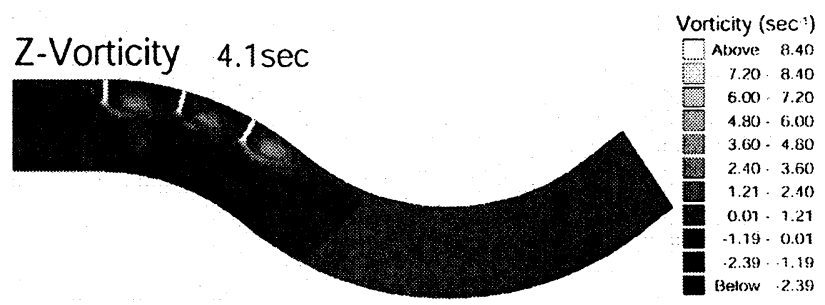

Fig.9 Vorticity plotted from calculation results

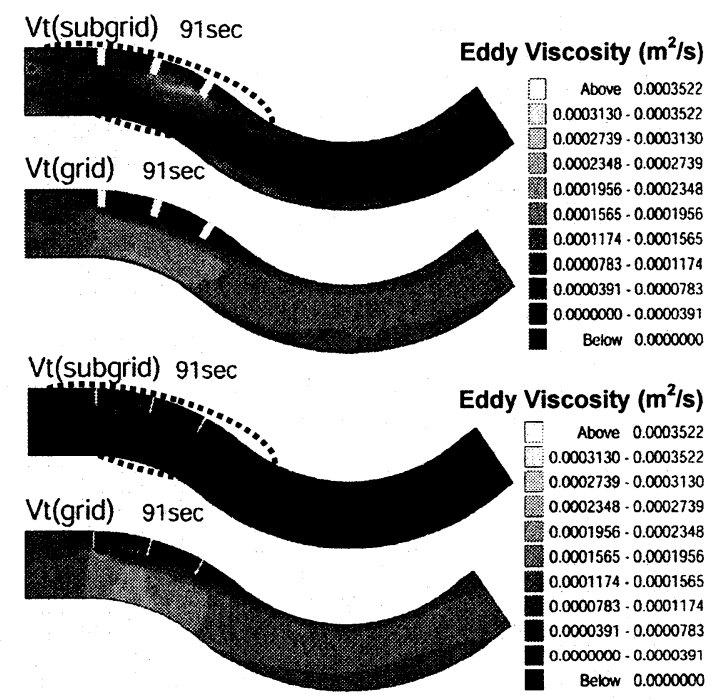

Fig.10 Eddy viscosity for "coarse" and "fine" mesh sizes

However, it is also noted that the difference has a small magnitude, perhaps it could be disregarded. This is because our grid size is relatively small $(2 \times 2 \mathrm{~cm})$ compared to water depth (about $10 \mathrm{~cm}$ ). Most of the eddy movement is already resolved in the grid scale. This makes the remaining unsolved eddy movement disappeared. The different grid sizes were then tested to verify the necessary of the SGS model to the turbulence. The next analysis is to investigate the effect of mesh size to the contribution of eddy viscosity. Compared to previously used mesh $(2 \times 2 \mathrm{~cm})$, "coarse $(5 \times 5 \mathrm{~cm})$ " and "fine $(1 \mathrm{x} 1 \mathrm{~cm})$ " meshes were generated.

It is obvious to see in Fig.10 that eddy viscosity calculates from SGS model is vanishing in the "fine" grid case. This means that the contribution to the eddy viscosity from SGS model is less as the grid size decreased. By striving at simulating the horizontal turbulent currents down to sufficiently 
small grid sizes we reduce the dependency of the results on the additional SGS model. Based on this, it would be very practical that if guideline for grid size selection with a certain level of necessity of using SGS for calculation model is provided. This could be one possibility to optimize the calculation efforts as it highly depends on mesh size.

\section{CONCLUSIONS}

This study confirmed that our available 2D model can perform a simulation with a good agreement to the existing experiment data set. Combined with LES for turbulence model by introducing SGS model to eddy simulations in 2D-turbulent flows, further improvement can be observed. Therefore, it can be concluded that the SGS model is successfully enhanced and suitable to our existing 2D model by modification of eddy viscosity due to energy dissipating into subgrid turbulence movement.

In modeling requirement point of view, among the flows which have less turbulence (quiet flow), the necessity of SGS to the model is becoming insignificant. It was found also that the larger scale of grid size in simulation (e.g. ocean scale), the more requirements of the SGS model because the magnitude of unresolved movements (eddy movement having a size smaller than calculation grid) is growing as the mesh size increases.

The benefit of SGS model is not only improvement of the calculation result, but also the calculation time as well, since the mesh size could be set larger, while achieving the same level of accuracy.

However, as descried, the contribution to the eddy viscosity from SGS model is less as the grid size decreases. By simulating the horizontal turbulent currents down to sufficiently small grid sizes, we reduce the dependency of the results on the additional SGS model. Then, to optimize the selection of grid size, another issue is raised that what would be the smallest grid size that the SGS contribution will be disappeared. If the "too fine" grid was selected, this will waste the calculation efforts. This problem is a challenge for our future work.

ACKNOWLEDGMENT: Authors would like to acknowledge for fruitful discussion and contribution of experimental data set from Sanjay Giri as well as
Rob Uittenbogaard (Delft Hydraulics) for worthy explanation on subgrid model.

\section{APPENDIX}

This appendix is devoted to explain about wall boundary condition applied here in this study. In momentum equation (2.2), the friction coefficient $\left(C_{d}\right)$ represents the shear force acting at the bottom for every grid location. Similarly at wall boundary, shear force has been additionally modeled by following definition.

$$
C_{d} u \sqrt{u^{2}+v^{2}} \Rightarrow C_{w} u \sqrt{u^{2}+v^{2}}
$$

Even though, the bed friction coefficient is based on manning formula, the wall friction is based on logarithmic velocity profile affected by wall shear friction. For this study, the wall friction coefficient $\mathrm{C}_{\mathrm{w}}$ was simply said to be 0.001 .

\section{REFERENCES}

1) Sadourny, R. \& Basdevant, C.: Parametrization of subgrid scale barotropic and baroclinic eddies in quasi-geostrophic models: Anticipated Potential Vorticity Method. Jour. Atmos. Sci., Vol.42, pp.1353-1363, 1985.

2) Jirka, Gerhard H.: Large Scale Flow Structures and Mixing Processes in Shallow Flows, Jour. of Hydraulic Research, Vol.39, No.6, pp.567-573, 2001.

3) Shimizu, Y.: Method for Simultaneous Computation of Bed and Bank Deformation of a River. In Bousmar \& Zech (eds.), River Flow 2002; Proc. of the Intern. Conf. on Fluvial Hydraulics, Louvain-la-Neuve, 4-6 September 2002. Belgium: A.A. Balkema Publishers.

4) Yabe, T. \& Ishikawa, T.: A Numerical Cubic-interpolated Pseudoparticle (CIP) Method without Time Splitting Technique for Hyperbolic Equations. Jour. of PSJ, Vol.59, No.7, pp.2301-2304, 1990.

5) Giri, S. \& Shimizu, Y.: Flow Characteristics in a Mildly Meandering Channel with \& without River Training Structures. Annual Jour. of Hyd. Eng., JSCE, Vol. 47, February 2003, pp.835-840, 2003.

6) Bendat, J. S. \& Piersol, A. G.: Random Data: Analysis \& Measurement Procedures, John Wiley \& Sons $3^{\text {rd }}$ edition, pp.297-298, 2000.

7) Uittenbogaard, R.: Summary SGS model for HLES. Note, Delft Hydraulics, 2001.

(Received September 30, 2003) 